\title{
Discussion on Stochastic Analysis of Hydraulic Vibration in Pressurized Water Diversion and Hydropower Systems
}

\author{
Jianxu Zhou * and Yu Chen \\ College of Water Conservancy and Hydropower Engineering, Hohai University, Nanjing 210098, China; \\ fluid_chan@hotmail.com \\ * Correspondence: jianxuzhou@163.com; Tel.: +86-138-518-93201
}

Received: 12 January 2018; Accepted: 20 March 2018; Published: 22 March 2018

\begin{abstract}
Hydraulic vibration exists in various water conveyance projects and has resulted in different operating problems, but its obvious effects on system's pressure head and stable operation have not been definitively addressed in the issued codes for engineering design, especially considering the uncertainties of hydraulic vibration. After detailed analysis of the randomness in hydraulic vibration and the commonly used stochastic approaches, in the basic equations for hydraulic vibration analysis, the random parameters and the formed stochastic equations were discussed for further probabilistic characteristic analysis of the random variables. Furthermore, preliminary investigation of the stochastic analysis of hydraulic vibration in pressurized pipelines and possible self-excited vibration in pumped-storage systems was presented for further consideration. The detailed discussion indicates that it is necessary to conduct further and systematic stochastic analysis of hydraulic vibration. Further, with the obtained frequencies and amplitudes in the form of a probability statement, the stochastic characteristics of various hydraulic vibrations can be investigated in detail and these solutions will be more reasonable for practical applications. Eventually, the stochastic analysis of hydraulic vibration will provide a basic premise to introduce its effect into the engineering design of water diversion and hydropower systems.
\end{abstract}

Keywords: stochastic analysis; hydraulic vibration; hydropower; stochastic equation; pressure pulsation

\section{Introduction}

Hydraulic vibration is a common phenomenon and exists in various water conveyance projects. Compared with water hammer, hydraulic vibration is a special and relatively severe hydraulic transient and has resulted in some operating problems including instabilities, local destruction, and even the interruption of services. For hydropower stations, because of the obvious interactions among the hydraulic system, the structural system, the mechanical system, and the electrical system, hydraulic vibration becomes more complex and has more effluence factors relative to different systems, but these significant effects on the pressure head and the evaluation of stable operation are also not definitively addressed in the issued codes for engineering design. Especially for the obvious uncertainties of hydraulic vibration, traditional deterministic analysis cannot fully reveal the inherent characteristics of existing hydraulic vibration; as a prudent measure, some reasonable suggestions for the effect of hydraulic vibration have been stated for the design of water diversion and hydropower systems, taking into consideration the difficulty of direct application of the obtained achievements in understanding hydraulic vibration [1]. Therefore, analysis of hydraulic vibration in water diversion and hydropower systems, which is more important and urgent than water hammer and surge analysis in most cases, should include stochastic analysis. 
Recently, probabilistic methodologies and stochastic analysis have been widely used in water and wastewater engineering, and have also played an important role in modern theories of nonlinear structural dynamics and the codes of structural design for hydraulic engineering (including hydropower systems). In the hydraulics of a hydraulic structure, stochastic approaches, such as statistical analysis, correlation analysis, and frequency-amplitude analysis, are widely applied to investigate some complex random hydraulic problems [2]. In particular, more stochastic models have been proposed and reasonably introduced to solve some stochastic problems in engineering design.

In a water distribution system, stochastic analysis is also considered as an important complement to deterministic analysis, not only in hydraulic transient control, but also in systematic optimization and demand model analysis. A new approach for reliability-based optimization of water distribution networks was presented to estimate network capacity reliability under random nodal demands and pipe roughness [3]. Based on a stochastic demand model and Monte Carlo simulation, the influence of cross-correlation and autocorrelation in demand on the probabilistic hydraulic performance of water networks was investigated [4]. To improve the pipe optimization under certain constraints, evaluation of the hydraulic reliability was achieved using Monte Carlo simulation and the Genetic Algorithm (GA) [5]. A decision tree was suggested to enumerate all possible valve failure combinations and corresponding event probabilities to evaluate system-wide valve failure impact on water distribution systems [6]. A methodology was presented to evaluate the impact of uncertainty in pipe roughness on decisions [7]. Based on field measurements, a real-time demand estimation method was presented for uncertainty quantification [8]. Duan et al. [9] pointed out that probabilistic analysis of hydraulic transients could be helpful for prudent design of new water supply systems and risk/reliability evaluation of existing systems. Liu et al. [10,11] provided a quantitative evaluation method for random pressure pulsation and external vibration of hydraulic pipelines based on reliability. A parameter estimation methodology for water distribution systems was proposed and the uncertainties in the estimated variables and resulting nodal pressure predictions were quantified in terms of confidence limits [12].

Stochastic approaches have also been successfully introduced into pulsating flow analysis in runner and draft tubes, and flow-induced vibrations, especially in hydropower stations. Like the flow-induced vibrations, the stability of some stochastic systems subjected to stochastic fluctuations was investigated and analytical expressions for the stochastic stability exponents were obtained with the Rayleigh Van Der Poi Oscillator; furthermore, the effects of parametric excitation components on stochastic response were also studied $[13,14]$. On the basis of the random vibration theory, the response spectrum method and advanced testing technology were used in the testing and calculation of large hydroelectric generating sets [15]. The dynamic characteristics of the stochastic hydro-turbine governing system were obtained from numerical experiments based on the simplified stochastic hydro-turbine governing model [16]. The dynamic interaction between the unsteady flow occurrence and the resulting vibration of the pipe was analyzed based on experiments and numerical models, and the importance of integrated analysis of fluid-structure interaction was then emphasized [17]. Based on the assumption of Poisson characteristics and application of stochastic modification of the second Lyapunov method for the pulsating fluid flow, the stability conditions of a pipeline section were analyzed [18]. Wu et al. [19] and Ohashi [20] comprehensively introduced the vibration and oscillation problems hazardous to the safety and reliable operation of hydraulic machinery and the problems caused by mechanical or hydraulic excitation, including self-excited vibration, along with the possible operational instability of the hydraulic, mechanical, structural, and power systems.

In hydropower stations, water hammer and water level fluctuation in surge tanks are all stochastic processes and their stochastic analysis is important for structural reliability design. For a reservoir-pipe-valve system, considering the initial head and wicket closing time as two random variables, the analytical probability distributions of the extreme value of water hammer were deduced from the approximate analytical formula [21,22]. With the built stochastic model for surge analysis of a typical hydraulic system with an upstream surge tank, the analytical probability distributions of 
several variables were revealed by Monte Carlo simulation [23]. Considering the initial load, wicket closing time, and reservoir's water level as random variables with their probability distributions, probability density formulas for water hammer were deduced and verified using case analysis [24]. Based on the established stochastic model of the water level in a surge tank, in which pipe roughness and original load were random variables, probability density functions of the head loss and maximum relative surge were deduced and further case analysis showed better agreement with results from the Monte Carlo method [25]. Finally, Zhang et al. [26] provided a preliminary exploration of a stochastic design for water hammer loading explicitly by considering various random parameters and conditions to advance the undertaking of reliability-based structural design.

Compared with water hammer and surge analysis in hydropower stations, because of the uncertainties of hydraulic disturbances, the complexity of vibration patterns and their formation mechanisms, and the random parameters, hydraulic vibration shows more complicated randomness. Zhang and Liu [27] mentioned that reliability design for impact vibration of hydraulic pressurized pipeline systems is still in the preliminary stages. Therefore, as a significant improvement to deterministic analysis, it is important and urgent to introduce stochastic approaches and probability theory into hydraulic vibration analysis. After a brief analysis of the random characteristics of hydraulic vibration and the basic stochastic approaches, the stochastic processing method for hydraulic vibration is introduced, and a preliminary investigation of the stochastic analysis of hydraulic vibration in pressurized pipelines and possible self-excited vibration in pumped-storage systems is then presented for further consideration. With the obtained frequencies and amplitudes in the form of a probability statement, the stochastic characteristics of various hydraulic vibrations can be investigated in detail, and these achievements will be contributory to practical applications.

\section{Randomness of Hydraulic Vibration}

Because of the obvious random characteristics of hydraulic vibration accompanied by random periodical pressure and flow oscillation, the stochastic analysis of hydraulic vibration is usually more complex than that of other hydraulic phenomena. In order to illustrate the complexity and uncertainties of hydraulic vibration intuitively, its effect on engineering design is conceptually analyzed; as the most important random parameter, the wave speed $a$ and its effect on hydraulic vibration analysis are further discussed.

For the structural design of the hydraulic system of a given hydropower station, the designed internal water pressure is a significant load on the pipe wall. According to conventional design, the maximum value of this load at each specified section consists of static pressure and pressure increase due to water hammer in either deterministic or stochastic analysis, regardless of the additional pressure produced by possible hydraulic vibration. In order to describe the effect of hydraulic vibration and its uncertainties, further analysis is conducted with a given simple pipe and hydraulic turbine system shown in Figure 1, in which Line 1 is the hydraulic gradient line (HGL), Line 2 is the computed maximum piezometric head line based on water hammer analysis, and Line 3 is the typical oscillatory curve for a specified hydraulic vibration mode.

Generally, for a pressurized pipe, because the maximum internal water pressure increases gradually along the water flow direction, the designed pipe thickness for the downstream part is always larger than that for the upstream part according to current design codes. Recently, based on stochastic analysis of water hammer and probability information of pressure increase [26], the internal water pressure and designed pipe thickness also follow the above distribution. Meanwhile, it is necessary to note that the water pressure induced by hydraulic vibration has a periodical distribution instead of a gradient curve, and the corresponding maximum pressure locates at some specified sections along the pressurized pipe. This means that, due to the possible severe hydraulic vibration, even in the upstream part of the pipe, the higher internal water pressure induced by hydraulic vibration may also exceed the designed pressure and lead to local structural damage. 


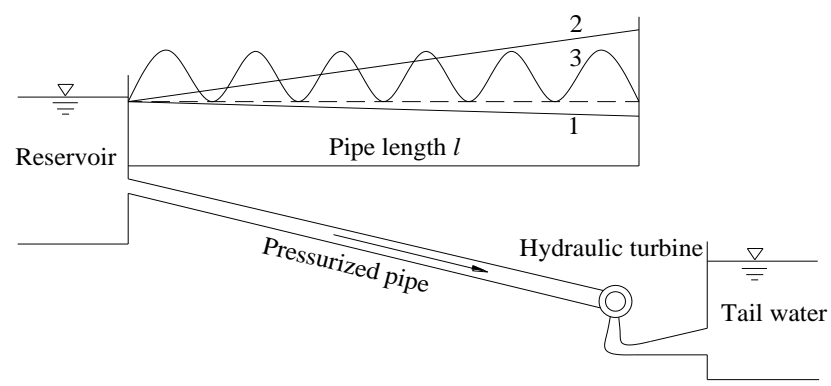

Figure 1. Conceptual distributions of different water pressures.

Furthermore, if the dynamic water pressure induced by possible hydraulic vibration is to be included in the structural design of the hydraulic system, the urgent problem is how to reasonably make this important inclusion given its distinct random characteristics and the deficiencies of deterministic analysis. Due to the variety and uncertainties of hydraulic vibration and possible disturbances in the hydropower stations, and the difficulty in fully and accurately evaluating the vibration characteristics and their effect on the structural design, there are also some key problems left for further investigation prior to the introduction of hydraulic vibration's effect into the engineering design. Before that, prudent consideration is necessary to guide engineering design based on detailed deterministic analysis and the accumulation of long-term practical experience. For example, to determine the designed maximum water pressure at the inlet of a spiral case and the minimum water pressure at the inlet of a draft tube, a necessary extra allowance is often required to cover the possible effect of pressure pulsation.

As a complex random phenomenon, the uncertainties of hydraulic vibration also include the random initial conditions, random boundary conditions, and some random parameters similar to other random processes. Most importantly, wave speed $a$ is a key random parameter in hydraulic vibration analysis, which could vary randomly due to the presence of uncertainties in a pressurized pipeline system, and the system's response to random frequencies is more sensitive to its variability than other random parameters. Other studies have focused on the complexity and randomness of the wave speed $a$. Suo and Wylie [28] presented a complex-valued and frequency-dependent wave speed in a rock-bored tunnel filled with water, and the theory of hydraulic resonance in pipelines was then extended to pressurized pipelines with frequency-dependent wave speeds. For water flow considering air content, Lee and Pejovic [29] pointed out that local wave speed variation is a function of the local transient air content and transient pressure. Covas et al. [30,31] also pointed out that wave speed is not a constant parameter in hydraulic transient analysis considering the different behavior of pipe materials and the effect of pipe wall viscoelasticity. Therefore, these studies confirmed that the wave speed $a$ is a complex random parameter especially for stochastic analysis of hydraulic vibration.

The complexity of the random wave speed and its essential effect on hydraulic vibration analysis can also be explained with a simple derivation. Assuming that the possible deviation of the wave speed is $\pm 10 \%$ with consideration of its uncertainties, and $k$ is a vibration mode of a simple hydropower system with pipe length $l$ shown in Figure 1 , the corresponding oscillation period $T_{k}$ is

$$
T_{k}=\frac{4 l}{a k} .
$$

Similarly, the corresponding oscillation period $T_{k+2}$ of the $k+2$ vibration mode is

$$
T_{k+2}=\frac{4 l}{a(k+2)} .
$$


With the preset wave speed deviation of $+10 \%$, the period of the $k$ vibration mode is supposed to be equal to that of the $k+2$ vibration mode, so

$$
\frac{4 l}{1.1 a k}=\frac{4 l}{a(k+2)} .
$$

The solution of (3) is easily obtained: $k=20$. This means that with $+10 \%$ error of the given wave speed $a$, the computed oscillation period of the 20-order vibration mode is equal to that of the 22-order vibration mode with the given wave speed $a$. This can be extended so that the higher natural frequencies may tend to be overlapped or misplaced due to uncertainties in the wave speed in a pressurized pipeline system. We can also conclude that the random wave speed has a great effect on the stochastic analysis of hydraulic vibration in pressurized water conveyance systems.

\section{Traditional Stochastic Approaches}

In the stochastic analysis of hydraulic problems like hydraulic vibration, if we regard some parameters as random variables according to their inherent characteristics, the specified hydraulic problems will be transferred into a random process, and, if possible, the obtained controlling differential equations can successfully be rewritten into stochastic differential equations combined with random variables and random processes.

Generally, there are two traditional methods to build the stochastic models [32]. The stochastic models are formed by introducing a Gaussian white noise process or Brownian motion into the original deterministic equations. In most cases, this type of stochastic differential equation often has analytical solutions as a Markov process and is widely used in applications. Sometimes, if the analytical solution is difficult to obtain explicitly, other optional approaches can be used to obtain the probability information of the solutions. The alternative to obtaining the stochastic equations is to regard the corresponding parameters in the original deterministic equations as random parameters. Usually, the analytical solution of this type of stochastic equation is complex and difficult to obtain, and the Liouville equation can be applied to derive the probability density function of the stochastic solutions. In some cases, if the analytical solution can be deduced for the corresponding deterministic equations, the stochastic solution is easily written with the same form as the deterministic one.

For these two stochastic models, the Monte Carlo method, which is more competitive in computer simulations and more practical in engineering applications, is a widely used simulation method for stochastic analysis. Kretzmann and Van Zyl [33] pointed out that stochastic analysis by Monte Carlo simulation can use any number of deterministic and stochastic parameters to describe different deterministic and probabilistic processes in the system, and the parameters used will largely depend on the purpose of the simulation and the original data. In addition, as a main compensatory stochastic analysis method for the Monte Carlo simulation method, the genetic algorithm is an optimization algorithm which mimics the biological evolution process in nature and has the ability to gain the optimum global solution at a fast convergence speed. Meanwhile, there are some other stochastic approaches, such as the Exponent Expansion method, the Runge-Kutta method, and the Random Euler Interpolation method.

In engineering, time domain analysis is always the main content of stochastic process simulation. With time domain analysis, more information including the time histories of oscillation amplitude and frequency can directly be obtained by nonlinear analysis, which is the premier method for hydraulic oscillation control. Meanwhile, frequency domain analysis is also an indispensable part, especially for stochastic analysis. The spectrum density function is a commonly used tool therein with the aim of obtaining its frequency characteristics and the required frequencies; power spectrum analysis can then be widely used for the stochastic analysis of hydraulic vibration. In the obtained power spectrum, both the possible range of main frequencies and further leading frequencies can be easily analyzed. Furthermore, Pan and Bu [34] introduced the Ensemble Empirical Mode Decomposition (EEMD) method for time-frequency analysis of the characteristics of pressure fluctuations. 


\section{Preliminary Investigation of Stochastic Analysis of Hydraulic Vibration}

\subsection{Stochastic Analysis of Hydraulic Pulsation}

As an important pattern of hydraulic vibration, stochastic pulsations including pressure pulsation and flow pulsation are the most common phenomena during the operation of hydropower stations, and possibly lead to dangerous flow-induced vibration. Hydraulic pulsation can be found all along the hydro-mechanical system, including the complex and hazard pressure oscillation in the draft tubes. Generally, the excited pressure pulsation comprises several vibration modes with different frequencies in both hydraulic models' test and prototype observations. There is no doubt that the pressure pulsation of water flow in pressurized water conveyance systems is a typical stochastic process and has some uncertainties, and some probabilities and statistical methods are often used to describe its quantitative characteristics. With a reasonable sampling time interval, sample size, record length, and repeated sampling number, the obtained sampling data should be analyzed by random data processing approaches on the basis of stochastic process theory. Eventually, the hydraulic characteristics of pressure pulsation should be presented by some probability indices, mainly including root mean square values, power spectrum, probability density, and correction function. Generally, there are two typical problems existing in the stochastic analysis of pressure pulsation.

One is to determine the stochastic fluctuation pressure according to the known power spectral density. Sometimes, the statistic sample can be re-examined on the basis of the frequency domain information using time domain simulation. With the possible or concerned natural frequencies of the pressure pulsation, the frequency range for the objective function can be defined; then, the time domain filter for the pressure pulsation can be obtained after the optimization of the filter's parameters by use of the genetic algorithm. The obtained filter is used to filter the random white noise and reasonable amplitude correction is then carried out according to the root mean square of the field data. Finally, the time history of the pressure pulsation can be re-examined for further control analysis.

The other is to conduct stochastic analysis for random pressure pulsation on the basis of the random data obtained by experimental research or field tests. Generally, necessary preprocessing should be applied for this original random data, including the elimination of the trend items, the conformation of the stationarity, and a digital filter [2]. Then, on the basis of the stationary stochastic processes without any trend items or invalid components, further probabilistic and statistical analysis can be performed with reasonable stochastic approaches.

The aim of statistical analysis is to obtain the probabilistic characteristics of the amplitudes and frequencies for the hydraulic pulsation. After statistical analysis, the probability distribution of the pressure pulsation is investigated, and then not only can the stochastic variables be described by the standard deviation, but also their values under different probabilities can be computed. In particular, on the basis of sample records and by use of power spectrum analysis, the power spectrum density distribution can be found, and both the possible range of main frequencies and existing leading frequencies can easily be analyzed.

\subsection{Stochastic Mathematical Model of Hydraulic Vibration}

In order to preliminarily investigate the stochastic problems in hydraulic vibration analysis, the stochastic mathematical model should first be built. Figure 2 shows a typical pipe segment with the given boundary conditions: the instantaneous piezometric head $H_{1}$ and flow rate $Q_{1}$ at the upstream end, and the instantaneous piezometric head $H_{2}$ and flow rate $Q_{2}$ at the downstream end.

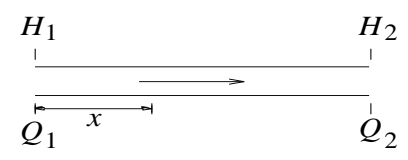

Figure 2. Pipe segment. 
The simplified basic equations for hydraulic transient analysis are also recommended on the basis of the given pipe segment in Figure 2, including the continuity equation and motion equation [35]

$$
\begin{gathered}
H_{x}+\frac{1}{g A} Q_{t}+\frac{f Q^{2}}{2 g D A^{2}}=0 \\
Q_{x}+\frac{g A}{a^{2}} H_{t}=0
\end{gathered}
$$

where $H$ and $Q$ are the piezometric head and flow rate in the time domain at any position $x$, respectively; $f, D, A$, and $a$ are the head loss coefficient, diameter, section area, and wave speed of the given pipe segment; subscript $x$ or $t$ means to derive with respect to $x$ or $t$, respectively; and $g$ is the acceleration of gravity.

For the steady state, the initial condition is

$$
H(x, 0)=H_{0}(x), Q(x, 0)=Q_{0}(x)
$$

where $H_{0}(x)$ and $Q_{0}(x)$ are the initial piezometric head and flow rate at a given section along the pipe. In hydraulic vibration analysis, either the natural boundary conditions or the essential boundary conditions are used. Here, the natural boundary condition is

$$
Q_{x}(0, t)=-\frac{g A}{a^{2}} \frac{d H_{1}(t)}{d t}, Q_{x}(l, t)=-\frac{g A}{a^{2}} \frac{d H_{2}(t)}{d t} .
$$

The essential boundary condition is

$$
Q(0, t)=Q_{1}(t), Q(l, t)=Q_{2}(t) .
$$

Obviously, in Equations (4) and (5), $f$ is a random parameter obtained from the random roughness $n$, which comprises the effect of local head loss, while another important random parameter is the wave speed $a$. All the others appear to be deterministic parameters. In the initial condition, $H_{0}(x)$ along the pipe is strongly related with the random roughness $n$ and the initial flow rate $Q_{0}(x)$; meanwhile, in the boundary condition, $H_{1}(t), H_{2}(t), Q_{1}(t)$, and $Q_{2}(t)$ are random variables. Therefore, if the random roughness $n$ and $a$ are mainly concerned, the basic equations for hydraulic vibration analysis are nonlinear and stochastic differential equations with random parameters, random initial conditions, and random boundary conditions.

Based on the stochastic theories, if the deterministic differential equations have analytical solutions, the analytical solutions for their stochastic equations can also be derived with the same solution forms. Otherwise, in order to obtain the statistical information of the concerned random variables, on the basis of the distributions of pipe roughness $n$ and wave speed $a$, the joint probability density function for the random variables in the stochastic differential equations should be deduced with necessary probabilistic information by use of the Liouville Equation [32].

According to above basic equations for hydraulic vibration analysis, the analytical solution for the deterministic differential equations can easily be deduced in the frequency domain. By means of linear approximation and Laplace transformation, Equations (4) and (5) can be transferred from time domain analysis to frequency domain analysis [35]:

$$
\begin{aligned}
& \frac{d H(s, x)}{d x}=-\gamma Z_{c} Q(s, x) \\
& \frac{d Q(s, x)}{d x}=-\frac{\gamma}{Z_{c}} H(s, x)
\end{aligned}
$$


where $H$ and $Q$ are piezometric head and flow rate in the frequency domain at $x$, respectively; $s$ is the Laplace variable; $\gamma$ is the propagation constant; and $Z_{c}$ is the characteristic impendence. $\gamma$ and $Z_{c}$ are dependent on $s$, and on the physical properties of the pipe flow and pipeline parameters.

Equations (9) and (10) are the basic equations to represent the hydraulic vibration characteristics of the water flow in pressurized pipelines, which is used for vibration mode analysis. For a given pipe segment with length $l$ (as in Figure 1), the piezometric head and flow rate in the frequency domain are also defined as $H_{1}$ and $Q_{1}$ for the upstream end, and $H_{2}$ and $Q_{2}$ for the downstream end; therefore, the natural boundary condition can be rewritten as follows:

$$
\left.\frac{d Q(s, x)}{d x}\right|_{x=0}=-\frac{\gamma}{Z_{c}} H_{1},\left.\frac{d Q(s, x)}{d x}\right|_{x=l}=-\frac{\gamma}{Z_{c}} H_{2}
$$

By taking Equation (10) as $H(s, x)=-\frac{Z_{c}}{\gamma} \frac{d Q(s, x)}{d x}$, substituting into Equation (9), and rearranging, the typical second-order ordinary differential equation is obtained.

$$
\frac{d^{2} Q(s, x)}{d x^{2}}-\gamma^{2} Q(s, x)=0
$$

On the basis of the assumption that $Q(x)=\sum_{i=0}^{n} q_{i} \cos \frac{i \pi x}{l}$ by linear combination with the oscillating flow rate $q_{i}$, and by use of the variation theory and Ritz-Galerkin approximation method, the analytical solution for problem (12) with natural boundary condition (11) can easily be derived [36,37]. The approximate solution for flow along the pipe is

$$
Q(x)=\frac{1}{l Z_{c} \gamma}\left(H_{1}-H_{2}\right)+\sum_{i=1}^{n} \frac{2 \gamma}{l Z_{c}} \frac{1}{\gamma^{2}+\left(\frac{i \pi}{l}\right)^{2}}\left[H_{1}-H_{2}(-1)^{i}\right] \cos \frac{i \pi x}{l} .
$$

For a simple pipe and turbine system, as shown in Figure 1, the flow at the downstream end of the pipe is often required. Then, the flow rate at the pipe's downstream end with $x=l$ is

$$
Q_{2}=Q(l)=\frac{1}{l Z_{c} \gamma}\left(H_{1}-H_{2}\right)+\sum_{i=1}^{n} \frac{2 \gamma}{l Z_{c}} \frac{1}{\gamma^{2}+\left(\frac{i \pi}{l}\right)^{2}}\left[H_{1}-H_{2}(-1)^{i}\right](-1)^{i} .
$$

After the expansion according to different orders and the introduction of the Laplace inverse transformation to each item, Equation (14) can be decomposed into a zero-order item $(I=0)$ and several $i$-order items.

The first-order differential equation for the zero-order flow rate obtained from the zero-order item, named the rigid model, is

$$
\frac{d q_{0}}{d t}+\frac{f Q_{0}}{D A} q_{0}=\frac{g A}{l}\left(\Delta H_{1}-\Delta H_{2}\right)
$$

The second-order differential equation for the $i$-order oscillatory flow rate obtained from each $i$-order item is

$$
\frac{d^{2} q_{i}}{d t^{2}}+\frac{f Q_{0}}{D A} \frac{d q_{i}}{d t}+\frac{a^{2}(i \pi)^{2}}{l^{2}} q_{i}=\frac{2 g A}{l}\left[\frac{d \Delta H_{1}}{d t}-\frac{d \Delta H_{2}}{d t}(-1)^{i}\right] .
$$

Defining the right items of Equations (15) and (16) as $f_{0}(x)$ and $f_{i}(x)$ respectively, they can be rewritten as follows:

$$
\begin{gathered}
\frac{d q_{0}}{d t}+\frac{f Q_{0}}{D A} q_{0}=f_{0}(t) \\
\frac{d^{2} q_{i}}{d t^{2}}+\frac{f Q_{0}}{D A} \frac{d q_{i}}{d t}+\frac{a^{2}(i \pi)^{2}}{l^{2}} q_{i}=f_{i}(t) .
\end{gathered}
$$


Equations (17) and (18) are typical first-order and second-order inhomogeneous ordinary differential equations, respectively. Given known $f_{0}(t)$ and $f_{i}(t)$, it is easy to derive the deterministic solutions of $q_{0}$ and oscillating flow rate $q_{i}(i=1,2, \ldots \ldots, n)$. Then, the deterministic solution of the flow rate variation at the downstream end of the pipe is

$$
\Delta Q_{2}(t)=\sum_{i=0}^{n} q_{i}(t)(-1)^{i}
$$

For the upstream pressurized pipe in Figure 1, because the water level of the upstream reservoir and the pressure oscillation at the downstream end are also random variables along with the random parameters-pipe roughness $n$ and wave speed $a$-each function $f_{i}(t)(I=0,1,2, \ldots \ldots, n)$ is obviously a stochastic process. Therefore, $q_{i}(i=0,1,2, \ldots \ldots, n)$ and $\Delta Q_{2}$ are random variables. With the known probabilistic distributions of all the random parameters and variables, and by introducing the mathematical expectation operator $\mathbf{E}$, the mean value of $\Delta Q_{2}$ has the same pattern as its deterministic solution, and it is written as

$$
\mathbf{E}\left[\Delta Q_{2}(t)\right]=\sum_{i=0}^{n} \mathbf{E}\left[q_{i}(t)\right](-1)^{i} .
$$

Similarly, the mean value of the other random variables can also be deduced under different boundary conditions. Therefore, for the stochastic analysis of hydraulic vibration of a simple pressurized pipe, the probability information of the possible random variables can basically be revealed by combination with deterministic analysis. On the basis of the above investigation, for the hydropower stations with a simple pipe and a turbine, the effect of the uncertain head flow characteristics of the hydraulic turbine, including possible pressure pulsation in the draft tube, can also be simplified and introduced into the random functions $f_{i}(t)(i=0,1,2, \ldots \ldots, n)$, so further stochastic analysis of hydraulic vibration in the hydropower stations can be investigated in detail.

\subsection{General Periodic Excitation with Random Disturbance}

As mentioned above, Equation (18) is a typical second-order inhomogeneous ordinary differential equation which describes a specified vibration mode of water flow in the pressurized pipe, and its right-hand side term can be viewed as an exciter. Generally, this exciter comprises some general periodic excitations. In practice, the periodic excitation may become disordered due to uncertainties in most cases. Therefore, if a specified vibration mode in stochastic analysis of hydraulic vibration is concerned, a stochastic model for general periodic excitations can be constructed by introducing a random disturbance to individual terms in the Fourier series of the corresponding deterministic periodic function, and the Monte Carlo method can be used to simulate different types of waveforms with random disturbances; then, with the possibly disordered periodic excitations, the nature of the excitation process can be revealed and further stochastic vibration can be analyzed in detail [38].

Let $f_{i}(t)$ be a basically periodic process with small random deviation from perfect periodicity; by adding random phase modulation to each term, $f_{i}(t)$ can be written as

$$
f_{i}(t)=\frac{a_{0}}{2}+\sum_{j=1}^{m}\left(a_{j} \cos \theta_{j}+b_{j} \sin \theta_{j}\right), \frac{d \theta_{j}(t)}{d t}=i \omega_{0}+\xi_{j}(t) .
$$

Then, Equation (18) can be written with a random Fourier series:

$$
\frac{d^{2} q_{i}}{d t^{2}}+\frac{f Q_{0}}{D A} \frac{d q_{i}}{d t}+\frac{a^{2}(i \pi)^{2}}{l^{2}} q_{i}=\frac{a_{0}}{2}+\sum_{j=1}^{m}\left(a_{j} \cos \theta_{j}+b_{j} \sin \theta_{j}\right)
$$

where $a_{0}, a_{j}, b_{j}(j=1,2, \ldots)$ are deterministic Fourier coefficients of the perfect periodic function; $\omega_{0}=2 \pi / T$, in which $T$ is the period of the corresponding undisturbed periodic function; $\theta_{j}$ is the phase angle of the given process; and $\xi_{j}(t)$ is a stationary and random white noise process with zero mean 
and intensity $D_{j}$. Random disturbances $\xi_{j}(t)$ of the $j$ th harmonic term $(j=1,2, \ldots)$ are assumed to be independent of each other.

In the case of no random phase disturbances $\left(D_{j}=0, j=1,2, \ldots\right), f_{i}(t)$ is simplified to a deterministic general periodic function with a period of $T$, and the response of the linear system to $f_{i}(t)$ can then be determined by the principle of superposition. Otherwise, on the basis of the above assumption of $\xi_{j}(t)$, the random Fourier series $f_{i}(t)$ is generally a non-zero-mean stationary and random process, which is convergent in the mean square sense.

Based on the Monte Carlo simulation method, the stochastic process of $f_{i}(t)$ can be produced with the consideration of the vibration characteristics and random components in the pressurized pipe. Meanwhile, by introducing some state variables, Equation (22) can be reformulated in the state space and transferred into a stochastic differential equation set which can be analyzed by the method of moments. Furthermore, by appropriately applying the Ito differential rule and the mathematical expectation operator $\mathbf{E}$, a set of deterministic equations for various orders of the response moments can be derived with all the obtained coefficients in the moment equations. Finally, the solutions can be computed for detailed analysis of different orders of moments of the response.

This model for stochastic analysis of hydraulic vibration is suitable for any vibration modes of water flow in pressurized pipes, and may be further developed to include other complex random disturbances. Furthermore, two important random parameters in Equation (22)-pipe roughness $n$ and wave speed $a$-may also be taken into consideration in this application.

\subsection{Stochastic Analysis of Self-Excited Vibration in Pump Turbine Systems}

Beside the hydraulic vibration in pressurized pipelines, self-excited vibration is also a typical problem in pumped-storage power stations. For the commonly used reversible-pump turbines, since the complete characteristic curves may have local S-shaped regions, pump turbines running or staying in these regions for a relatively long time may undergo possible self-excited oscillation. During the operation of pumped-storage power stations, most of the reported oscillation problems are closely associated with the unstable local S-shaped regions in the complete characteristic curves of pump turbines [39].

For single-pump-turbine systems and two-pump-turbine systems with symmetry branches either in turbine operation or in pump operation, the self-excited oscillation equations are consistent in form and the uniform equation [39] can be written as

$$
\frac{d^{2} q}{d t^{2}}-\varepsilon \frac{d q}{d t}\left(1+\sigma q+\delta q^{2}\right)+\omega_{1}^{2} q=0
$$

where $q$ is the oscillating flow rate of each pump turbine; $\omega_{1}$ is the first-order natural angular frequency of different dynamic systems; and $\varepsilon, \sigma$, and $\delta$ are the known coefficients concerned with the specified head flow relation in the S-shaped region, initial flow of the pump turbine, and pipe parameters including cross-sectional area, pipe length, and head loss coefficient.

Generally, $\varepsilon$ is a smaller positive constant which is the basic premise to the possibility of negative damping and the instability in the S-shaped regions, and $\delta$ is a negative constant with smaller absolute value; therefore, Equation (23) describes the self-excited oscillation of a weakly nonlinear autonomous system. For deterministic analysis, the second-degree approximate analytical solution of Equation (23) [39] is

$$
q(t)=B \cos \left(\omega_{1} t+\theta_{1}\right)+\sum_{k=2}^{5} B_{m k} \sin \left(k \omega_{1} t+\varphi_{k}\right)+\frac{\varepsilon^{2} \sigma B^{2}}{8 \omega_{1}^{2}}\left(4+\delta B^{2}\right)
$$

where $B$ is the amplitude of the first-order oscillating flow rate, $B(t)=\frac{2}{\sqrt{\left(4 / B_{0}^{2}+\delta\right) e^{-\varepsilon t}-\delta}} ; B_{0}$ is the initial amplitude of the disturbance; $B_{m k}(k=2,3,4,5)$ is the amplitude of the $k$ th oscillation; $\theta_{1}$ is the phase angle of the first oscillation; and $\varphi_{k}(k=2,3,4,5)$ is the phase angle of the $k$ th oscillation. 
Obviously, the S-shaped regions of the reversible-pump turbines usually have some uncertain factors and it is rather difficult to illustrate these characteristics in detail by experimental research or numerical simulation. While sensitivity analysis is often required for some uncertain parameters in deterministic analysis, stochastic analysis is also recommended for the self-excited vibration. With the known probabilistic distributions of all the random parameters, and by introducing the mathematical expectation operator $\mathbf{E}$, the mean value of the random variable $q$ has the same pattern as the obtained deterministic solution given in Equation (24).

$$
\mathbf{E}[q(t)]=\mathbf{E}\left[B \cos \left(\omega_{1} t+\theta_{1}\right)\right]+\sum_{k=2}^{5} \mathbf{E}\left[B_{m k} \sin \left(k \omega_{1} t+\phi_{k}\right)\right]+\mathbf{E}\left[\frac{\varepsilon^{2} \sigma B^{2}}{2 \omega_{1}^{2}}\right]+\mathbf{E}\left[\frac{\varepsilon^{2} \sigma \delta B^{4}}{8 \omega_{1}^{2}}\right]
$$

In particular, it can also be inferred from (24) that when $t \rightarrow \infty$ and $\delta<0$, as long as $B_{0} \neq 0$, the amplitude $B$ will tend to a steady value of $2(-\delta)^{-0.5}$, which is independent from initial conditions including the value of $B_{0}$. Defining the head flow relation in the S-shaped region of the pump turbine as $H=c_{3} Q^{3}+c_{2} Q^{2}+c_{1} Q+c_{0}$, in which $c_{i}(i=0,1,2,3)$ are random coefficients approximately deduced from the flow characteristic in the local S-shaped region, then the random parameter $\delta$ can be represented as a function of some other random parameters.

$$
\delta=f\left(Q_{0}, \alpha, c_{3}, c_{2}, c_{1}, c_{0}\right)
$$

Similarly, based on its deterministic solution, the mean value of the random variable $B$ is

$$
\mathbf{E}[B(t)]=\frac{2}{\mathbf{E}[\sqrt{-\delta}]}
$$

Equation (27) means that with the premise that the probabilistic distribution of the random parameter $\delta$ is known on the basis of careful analysis, the probabilistic information of the maximum amplitude of the self-excited vibration can be computed for further analysis. With the stochastic analysis of the self-excited vibration, the effect of the S-shaped regions of pump turbines on operating stability can be revealed clearly and completely.

\section{Conclusions}

Hydraulic vibration is a relatively severe hydraulic transient phenomenon and exists in various water conveyance projects. For hydropower stations, the effects of hydraulic vibration on the pressure head and the evaluation of stable operation are not clearly addressed in the issued codes for engineering design, mainly due to its obvious randomness. Therefore, stochastic approaches and probability analysis should be introduced into hydraulic vibration analysis.

After a brief analysis of the random characteristics of hydraulic vibration and the basic stochastic approaches, a stochastic processing method for the hydraulic pulsation was introduced, and a preliminary investigation of the stochastic analysis of the hydraulic vibration in the pressurized pipelines was then presented on the basis of hydraulic vibration theory and deterministic solutions. In particular, for a given general periodic excitation with random disturbance acting on a specified vibration mode of water flow in a pressurized pipe, the system's stochastic response analysis by means of the Monte Carlo simulation method was discussed. Furthermore, possible self-excited vibration in pumped-storage power stations was also introduced with stochastic approaches.

Based on our preliminary investigation and detailed discussion of the stochastic analysis of hydraulic vibration in water diversion and hydropower systems, we conclude that the reasonable probabilistic distributions of some random parameters, such as pipe roughness $n$ and wave speed $a$, should be analyzed through detailed investigation, and their probability indices mainly including the expected values and variances should be deduced. Comprehensive and systematic stochastic analysis should then be implemented to solve different hydraulic vibration problems including hydraulic 
characteristic analysis, hydraulic disturbance analysis, dynamic response analysis, and self-excited vibration analysis, etc. With the obtained frequencies and amplitudes in the form of a probability statement, the stochastic characteristics of various hydraulic vibrations can be revealed in detail; this will be more reasonable for practical applications. Eventually, with progress in the stochastic analysis of hydraulic vibration, the effect of hydraulic vibration can be reasonably considered in the future engineering design of water diversion and hydropower systems.

Acknowledgments: The authors gratefully acknowledge support from the Projects Funded by the National Natural Science Foundation of China (Grant Nos. 51079051 and 51479071), the National Key R\&D Program of China (No. 2016YFC0402501), and the Priority Academic Program Development of Jiangsu Higher Education Institutions (PAPD, SYS1401).

Author Contributions: Jianxu Zhou initiated and completed the literature review and discussion, and then drafted the literature search, figure editing, study design, data analysis, data interpretation, and writing, etc. Yu Chen prepared the references, and edited and revised the literature search, data analysis, and writing, etc.

Conflicts of Interest: The authors declare no conflict of interest.

\section{References}

1. Li, X.S.; Gao, Y.; Dong, X.B. Discussion on the design of regulation guarantee of hydropower stations. Water Power 2014, 40, 58-60.

2. Ding, Z.Y.; Zhou, C. Basis of Stochastic Approaches and Their Application in the Hydraulic Engineering and Hydraulics; Changjiang Press: Wuhan, China, 2005; ISBN 978-7-80708-019-0.

3. Tolson, B.A.; Maier, H.R.; Simpson, A.R.; Lence, B.J. Genetic algorithms for reliability-based optimization of water distribution systems. J. Water Resour. Plan. Manag. 2004, 130, 63-72. [CrossRef]

4. Filion, Y.R.; Karney, B.W.; Adams, B.J. Stochasticity of demand and probabilistic performance of water networks. In Proceedings of the ASCE World Water and Environmental Resources Congress, Anchorage, AK, USA, 15-19 May 2005.

5. Djebedjian, B. Reliability-based water network optimization for steady state flow and water hammer. In Proceedings of the ASME 6th International Pipeline Conference, Calgary, AB, Canada, 25-29 September 2006.

6. Jun, H.; Loganathan, G.V.; Deb, A.K.; Grayman, W.; Snyder, J. Valve distribution and Impact analysis in water distribution systems. J. Environ. Eng. 2007, 133, 790-799. [CrossRef]

7. Sumer, D.; Lansey, K. Effect of uncertainty on water distribution system model design decisions. J. Water Resour. Plan. Manag. 2009, 135, 38-47. [CrossRef]

8. Kang, D.; Lansey, K. Real-time demand estimation and confidence limit analysis for water distribution systems. J. Hydraul. Eng. 2009, 135, 825-837. [CrossRef]

9. Duan, H.F.; Tung, Y.K.; Ghidaoui, M.S. Probabilistic analysis of transient design for water supply systems. J. Water Resour. Plan. Manag. 2010, 136, 678-687. [CrossRef]

10. Liu, W.; Liu, Y.S.; Yue, Z.F. Dynamic reliability of aircraft hydraulic pipelines under random pressure pulsation and vibration. Multidiscip. Model. Mater. Struct. 2010, 6, 493-507. [CrossRef]

11. Liu, W.; Liu, Y.S.; Jiang, Z.F.; Yue, Z.F. Pressure pulsation reliability analysis of hydraulic power pipelines. J. Vib. Shock 2011, 30, 265-268.

12. Kang, D.; Lansey, K. Demand and roughness estimation in water distribution systems. J. Water Resour. Plan. Manag. 2011, 137, 20-30. [CrossRef]

13. Jedrzejewski, F. Stochastic stability of coupled dynamical systems. In Proceedings of the ASME Pressure Vessels and Piping Conference, Atlanta, GA, USA, 22-26 July 2001; pp. 147-154.

14. Jedrzejewski, F. Stochastic stability of some Hamiltonian systems. In Proceedings of the ASME of Pressure Vessels and Piping Conference, Vancouver, BC, Canada, 5-9 August 2002; pp. 187-191.

15. Xiao, L. Random vibrations' analysis of hydroelectric generating set. J. Yangtze River Sci. Res. Inst. 2009, $26,62-65$.

16. Xu, B.B.; Chen, D.Y.; Tolo, S.; Patelli, E.; Jiang, Y.L. Model validation and stochastic stability of a hydro-turbine governing system under hydraulic excitations. Electr. Power Energy Syst. 2018, 95, 156-165. [CrossRef]

17. Simão, M.; Mora-Rodriguez, J.; Ramos, H.M. Mechanical interaction in pressurized pipe systems: Experiments and Numerical Models. Water 2015, 7, 6321-6350. [CrossRef] 
18. Carkovs, J.; Matvejevs, A.; Pavlenko, O. Stochastic stability of a pipeline affected by pulsate fluid flow. Procedia Comp. Sci. 2017, 104, 12-19. [CrossRef]

19. Wu, Y.L.; Li, S.C.; Liu, S.H.; Dou, H.S.; Qian, Z.D. Vibration of Hydraulic Machinery; Springer: Dordrecht/Heidelberg, Germany; New York, NY, USA, 2013; ISBN 978-94-007-6421-7.

20. Ohashi, H. Vibration and Oscillation of Hydraulic Machinery; Routledge, Taylor and Francis Group: New York, NY, USA, 2016; ISBN 978-1-85628-185-0.

21. Guo, W.Z.; Suo, L.S. Stochastic analysis of water hammer in single pipe. J. Hydroelectr. Eng. 1996, $15,72-81$.

22. Zhang, Q.F.; Suo, L.S.; Guo, W.Z. A further study of stochastic analysis of water hammer pressure in a reservoir-pipe-valve system. J. Hydroelectr. Eng. 2000, 19, 56-63.

23. Zhang, Q.F.; Suo, L.S. Stochastic model for surge analysis. J. Hohai Univ. 1997, 25, 41-45.

24. Zhu, Y.Z.; Zhang, J.; Hu, M. Random model of water hammer pressure and probability analysis in waterpower station. In Proceedings of the ASME 5th Joint ASME/JSME Fluids Engineering Summer Conference, San Diego, CA, USA, 30 July-2 August 2007; Volume 2, pp. 47-55.

25. Zhu, Y.Z.; Zhang, J.; Yuan, Y.S.; Chen, J.R.; Zheng, Y. The probability distribution of the relative highest water level in surge tank of waterpower station. In Proceedings of the ASME Fluids Engineering Division Summer Conference, Jackonsville, FL, USA, 10-14 August 2008; Volume 2, pp. 375-379.

26. Zhang, K.Q.; Karney, B.W.; Suo, L.S.; Colombo, A.F. Stochastic analysis of water hammer and applications in reliability-based structural design for hydro turbine penstocks. J. Hydraul. Eng. 2011, 137, 1509-1521. [CrossRef]

27. Zhang, T.X.; Liu, X.H. Reliability design for impact vibration of hydraulic pressure pipeline systems. Chin. J. Mech. Eng. 2013, 26, 1050-1055. [CrossRef]

28. Suo, L.S.; Wylie, E.B. Hydraulic transients in rock-bored tunnels. J. Hydraul. Eng. 1990, 116, 196-210. [CrossRef]

29. Lee, T.S.; Pejovic, S. Air influence on similarity of hydraulic transients and vibrations. J. Fluids Eng. 1996, 118, 706-709. [CrossRef]

30. Covas, D.; Stoianov, I.; Mano, J.F.; Ramos, H.; Graham, N.; Maksimovic, C. The dynamic effect of pipe-wall viscoelasticity in hydraulic transients. Part I-Experimental analysis and creep characterization. J. Hydraul. Res. 2004, 42, 517-532. [CrossRef]

31. Covas, D.; Stoianov, I.; Mano, J.F.; Ramos, H.; Graham, N.; Maksimovic, C. The dynamic effect of pipe-wall viscoelasticity in hydraulic transients. Part II-Model development, calibration and verification. J. Hydraul. Res. 2005, 43, 56-70. [CrossRef]

32. Zhu, Y.Z. Random Model and Solution to Surge and Water Hammer. Ph.D. Thesis, Hohai University, Nanjing, China, 2005.

33. Kretzmann, H.A.; Van Zyl, J.E. Stochastic analysis of water distribution systems. In Proceedings of the ASCE World Water and Environmental Resources Congress, Salt Lake City, UT, USA, 27 June-1 July 2004.

34. Pan, H.; Bu, M.S. Pressure fluctuation signal analysis of pump based on ensemble empirical mode decomposition method. Water Sci. Eng. 2014, 7, 227-235. [CrossRef]

35. Wylie, E.B.; Streeter, V.L.; Suo, L.S. Chapter 12: Concepts of Oscillatory Flow and Resonance; and Chapter 13: Analysis of Oscillatory Flow in Systems. In Fluid Transients in Systems; Prentice-Hall, Englewood Cliffs: Upper Saddle River, NJ, USA, 1993; ISBN 978-0-13934-423-7.

36. Zhou, J.X.; Suo, L.S.; Zhu, Y.Z. Study on elastic model of water flow in pressurized pipeline based on variation theory. J. Hydroelectr. Eng. 2008, 27, 109-113.

37. Zhou, J.X.; Cai, F.L.; Wang, Y. New elastic model of pipe flow for stability analysis of the governor-turbine-hydraulic system. J. Hydroelectr. Eng. 2011, 137, 1238-1247. [CrossRef]

38. Zhou, Y.S.; Hou, Z.K.; Dimentberg, M.F.; Noori, M.N. A model for general periodic excitation with random disturbance and its application. J. Sound Vib. 1997, 203, 607-620. [CrossRef]

39. Zhou, J.X.; Karney, B.W.; Hu, M.; Xu, J.C. Analytical study on possible self-excited oscillation in S-shaped regions of pump-turbines. Proc. Ins. Mech. Eng. Part A J. Power Energy 2011, 225, 1142. [CrossRef]

(C) 2018 by the authors. Licensee MDPI, Basel, Switzerland. This article is an open access article distributed under the terms and conditions of the Creative Commons Attribution (CC BY) license (http:/ / creativecommons.org/licenses/by/4.0/). 